\title{
Asymptotic Theory of Second Order Differential Equations with Two Simple Turning Points
}

\author{
Nicholas D. KaZarinofF \\ Communicated by L. CESARI
}

1. Introduction ${ }^{1}$. In 1931 Langer initiated and gave the first of numerous contributions to what has become a successful theory for asymptotic expansions of the solutions of a differential equation with a turning point. This theory has been extended and applied to a great many questions by him and by others. An extensive list of references may be found in L. CESARI's book, Asymptotic behavior and stability of solutions of differential equations, Chapter IV, SpringerVerlag, Berlin, 1958.

In this paper we present a general asymptotic theory of ordinary second order linear differential equations with two simple turning points and containing a numerically large parameter. In particular, we are concerned with the asymptotic expansions with respect to complex $\lambda$ of solutions of differential equations of the form

$$
\frac{d^{2} y}{d s^{2}}-\lambda^{2} P(s, \lambda) y=0 \text {. }
$$

We consider this equation for $s$ in a closed, simply connected, perhaps unbounded region $\mathscr{D}_{s}$ of the complex plane. We assume that for $|\lambda|>N$ and for $s \in \mathscr{D}_{s}$ the coefficient $P(s, \lambda)$ is of the form ${ }^{2}$

$$
P(s, \lambda)=\sum_{0}^{\infty} p_{j}(s) \lambda=1,
$$

where each $p_{j}$ is analytic, and, most importantly, that $p_{0}(s)$ has precisely two simple zeros, $\alpha$ and $\beta$, in the interior of $\mathscr{D}_{s}$.

In a region which includes a turning point, i.e., a zero or singularity of $p_{0}(s)$, the solutions of the differential equation (1.1) depend upon $\lambda$ in so intricate a way as to have quite distinct asymptotic forms in different parts of the region, being dominant (exponentially large) in some parts and subdominant (exponentially small) in others. The asymptotic series for solutions of (1.1) over a region

1 This research was supported by the United States Air Force through the Air Force Office of Scientific Research of the Air Research and Development Command under contracts No. AF 18(600)-1481 and No. AF 49(638)-192.

2 The letters $M$ and $N$ are always to be used as generic symbols for positive constants. 
which includes just one simple turning point are known and are based upon Airy functions $[2,5]$. Thus, it would appear that the behavior of solutions of (1.) over the entire region $\mathscr{D}_{s}$, which contains two simple turning points, is obtainable by the familiar procedure of evaluating the coefficients in the dependence relations which connect solutions whose behavior is known about one of the turning points with those solutions whose behavior is known about the other turning point. Unfortunately, this evaluation is not possible in general; two liiearly independent solutions may have the same dominant asymptotic form, which makes the inference of the identity of two solutions from the identity of their asymptotic forms invalid. Thus, a new theory is necessary if we desire to have uniform asymptotic expansions of solutions of (1.1) over all $\mathscr{D}_{s}$. LANGER [4] has derived the leading terms of such asymptotic expansions in the special case where $s$ is a real variable on a bounded interval, $p_{0}(s)$ is real-valued, and $p_{1}(s) \equiv 0$. We derive the asymptotic expansions to $n+1$ terms, where $n$ is an arbitrary non-negative integer, of the solutions of (1.1) under the general hypotheses set forth in the first paragraph above together with some others of a more technical nature to be set forth later.

Interest in the problem discussed here stems mainly from possible applications for the theory derived. In certain regions, the differential equations for the angular and radial spheroidal functions are of the type (1.1). This is also true of the Whittaker equation for certain configurations of its parameters. The spheroidal functions are important in problems concerning scattering by a prolate spheroid. The Whittaker functions, disguised as Coulomb wave functions, occur in quantum mechanics. Equations of type (1.1) are also of interest in other problems of wave motion and diffraction.

We have divided the discussion below into three parts. In Part I we transform the differential equation (1.1) into one more suitable for analysis. We call this canonical form the given equation. We then give an algorithm for the construction of a related equation whose coefficients resemble the coefficients of the given equation to an arbitrarily prescribed degree. In Part II we study the solutions of the related equation. These involve Weber functions of large complex order and argument. They have been studied by ERDÉLYI, KENNEDY \& MCGREGOR [3]. iVe make considerable use of their results and have shown that their asymptotic representations hold uniformly in $\arg v$ over a finite range of $\arg v$, where $v$ is the order of the Weber functions involved. We also give an algorithm for recursively determining the terms in the asymptotic expansions of these Weber functions. In Part III we prove that solutions of the related equation are asymptotic expansions to $n+1$ terms of solutions of the given equation, $n$ being any non-negative integer. The method of proof is, as usual, to transform the given equation into an integral equation of Volterra type, whose kernel involves solutions of the related equation, and to solve this integral equation by the Picard method of successive iteration. We also give approximations for derivatives of solutions of the given equation. Our main results are stated as Theorems 1 and 2 of $\S \S 12$ and 13. An especially interesting feature is the occurence of a denumerable number of characteristic solutions of the given equation. These are bounded and oscillatory on certain curves joining the turning points and exponentially small on their extensions beyond the turning points: 


\section{Part I: Construction of the Related Equation}

2. The given equation. The analysis to follow involves certain functions and a mapping connected with $p_{0}(s)$. To simplify their form we adopt a normalized form of the differential equation (1.1) as the basis of the sequel. This normalization was used by LANGER in $[4, \S \S 2,3]$. Its adoption here will be seen to entail certain assumptions on $p_{0}(s)$.

We consider the mapping from the given region $\mathscr{D}_{s}$ onto a region $\mathscr{D}_{z}$ of the $z$-plane defined implicitly by the equation

$$
\int_{+1}^{z}\left(t^{2}-1\right)^{\frac{1}{3}} d t-\int_{\alpha}^{s} p_{0}^{5}(t) d t=0
$$

An immediate question is whether or not (2.1) defines a mapping at all, and if so, is it a schlicht mapping? We can show that (2.1) defines a mapping and that at each point of $\mathscr{D}_{s}$ it defines a locally one-to-one mapping. The function which is the left member of (2.1) is analytic in $z$ except at \pm 1 and in $s$ except at $\alpha$ and $\beta$. Its partial derivatives do not vanish except on the lines $z= \pm 1$ and $s=\alpha$ or $\beta$. Thus by the implicit function theorem for analytic functions, there exist in neighborhoods of all points of $\mathscr{D}_{s}$, except possibly $\alpha$ and $\beta$, analytic solutions $z(s)$ of (2.1) with inverses $s(z)$. Further, $d s / d z=\left(z^{2}-1\right)^{\frac{1}{2}} p_{0}^{-\frac{1}{2}}(s)$; and hence, $s^{\prime}(z) \neq 0$ except perhaps at \pm 1 .

We now examine what happens at the exceptional points $\alpha$ and $\beta$. A computation shows that we may write

$$
\int_{1}^{z}\left(t^{2}-1\right)^{\frac{1}{2}} d t=(z-1)^{\frac{3}{2}} \Phi_{1}(z) \text { and } \int_{\alpha}^{s} p_{0}^{\frac{1}{2}}(t) d t=(s-\alpha)^{\frac{3}{2}} P_{1}(s),
$$

where $\Phi_{1}$ and $P_{1}$ are analytic in neighborhoods of +1 and $\alpha$, respectively, and neither $\Phi_{1}(1)$ nor $P_{1}(\alpha)$ is zero. Thus, we may write

$$
\dot{F}(z, s)=(z-1) \Phi_{1}^{q}(z)-(s-\alpha) P_{1}^{q}(s)=0,
$$

where $F$ is analytic in a neighborhood of $(1, \alpha)$ and neither $\partial F / \partial z$ nor $\partial F / \partial s$ vanishes at $(1, \alpha)$. Therefore, by the implicit function theorem, there exists a solution $z(s)$ of (2.3), and hence of (2.1), with inverse $s(z)$, which is analytic in a neighborhood of $\alpha$ and such that $z(\alpha)=1$ and $s^{\prime}(1) \neq 0$.

In order to apply this same technique to $(2.1)$ at $s=\beta$, we must assume . normalization of the coefficient $p_{0}$. The integrals $\int_{1}^{-1}\left(t^{2}-1\right)^{\frac{1}{t}} d t$ and $\int_{\alpha}^{\beta} p_{0}^{t}(t) d t$ -are independent of path provided the pachs do not encircle the singularities of the integrands. However, it may be that the latter integral vanishes. We assume the contrary and choose the parameter so that ${ }^{3}$

$$
\int_{\alpha}^{\beta} p_{0}^{\frac{1}{2}}(t) d t=\int_{1}^{-1}\left(t^{2}-1\right)^{\frac{1}{2}} d t=-\frac{1}{2} \pi i .
$$

We can now apply considerations analogous to those above to reach the conclusions that $(2.1)$ has an analytic solution $z(s)$ at $(-1, \beta)$ with inverse $s(z)$

${ }^{a}$ We do not consider the limiting behavior of the solutions of (1.1) as the turning points $a$ and $\beta$ approach each other. F. W. J. OLVER has informed the author by a private communication that he is examining this limiting behavior. 
whose derivative $s^{\prime}$ is nonzero at -1 . If $\arg p_{0}$ is constant on the line segment joining $\alpha$ and $\beta$, the hypothesis (i) is clearly fulfilled. This is the case treated by LANGER [4]; and since, in this instance, the mapping defined by (2.1) is one to one on the line segment joining $\alpha$ to $\beta$, it is schlicht in an open neighborhood of this closed segment.

We have shown that the implicit relation (2.1) defines a mapping from $\mathscr{D}_{s}$ onto a region $\mathscr{D}_{z}$ and that it defines a locally one to one mapping of some neighborhood of any point of $\mathscr{D}_{s}$ onto a neighborhood of $\mathscr{D}_{z}$. We require more than this and assume that

(ii) The relation (2.1) defines a schlicht mapping

with $[-1,1]<\mathscr{D}_{\mathrm{z}}$.

$$
z(s): \mathscr{D}_{s} \leftrightarrow \mathscr{D}_{z},
$$

Under this assumption, the change of variables

$$
\begin{aligned}
s & =s(z), \\
y(s) & =\left\{\frac{d s}{d z}\right\}^{\frac{1}{2}} u(z),
\end{aligned}
$$

where $s$ satisfies (2.1), transforms the differential equation (1.1) into

$$
\frac{d^{2} u}{d z^{2}}-\lambda^{2} Q(z, \lambda) u=0
$$

with

$$
Q(z, \lambda)=P(s(z), \lambda) s^{2}+\frac{1}{\lambda^{2}}\left\{\frac{3 s^{\prime \prime 2}}{4 s^{\prime 2}}-\frac{s^{\prime \prime \prime}}{2 s^{\prime}}\right\} \quad\left(\prime=\frac{d}{d z}\right)
$$

where $Q$ is analytic for $|\lambda|>N$ and for $z \in \mathscr{D}$. We write

In particular,

$$
Q(z, \lambda)=\sum_{0}^{\infty} q_{j}(z) \lambda^{-i}
$$

$$
q_{0}(z)=\left(z^{2}-1\right)
$$

We henceforward refer to the differential equation (2.5) as "the given equation".

3. The first approximating equation. The analysis of the given equation is based upon the construction of an equation which resembles it up to terms of the form $\lambda^{-n-1} O_{(1)}$ in the coefficient of $u$, where $n$ is any non-negative integer and $O(1)$ denotes a function of $z$ and $\lambda$ which is bounded for $z \in \mathscr{D}_{z}$ and for $|\lambda|>N$. The algorithm for the construction of this related equation is similar to the algorithm of McKelvey given in [6]. The matter at issue in McKelvey's paper is the approximation of solutions of an equation with a single turning point of order two. Such a turning point may be thought of as the confluence of two simple turning points so that similarities in the analyses of the two cases are not unexpected.

The point of beginning in both instances is WEBER's equation, which may be written in the form

$$
\frac{d^{2} V^{r}}{d t^{2}}+\left[\frac{v}{2}-\frac{t^{2}}{4}\right] V=0
$$


We make the transformation

$$
t=(2 \lambda)^{!} z, \quad V(t)=v(z),
$$

and Weber's equation becomes

$$
d z^{2}-\lambda\left(\lambda z^{2}-v\right) v=0
$$

Let a non-negative integer $n$ be chosen once for all. We now choose $y^{\prime}$ so that

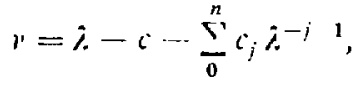

where the (arbitrary) constants $c$ and $c_{j}$ are yet to be determined. This brings IVEBER's equation into the form

$$
d^{2} z^{2}-\left[z^{2}\left(z^{2}-1\right)-\lambda i+\frac{\sum_{0}^{\prime}}{n} c_{j} \lambda \mid i=0 \text { or } i^{\prime \prime}-R(z, \lambda) z^{\prime}=0\right.
$$

which is our first approximating equation.

4. The second approximating equation. We next construct an equation resembling the given equation in both the $\lambda^{2}$ and $\lambda$ terms of the coefficient of $u$. Formally, this step i: almost identical with the corresponding one in $[6]$. The idea is to make a change if dependent variable

$$
Z=\left\|_{0} \hat{\imath}^{\prime}+\right\|_{1} \tilde{i}^{\prime} / \hat{\lambda}
$$

in the first approximating equation (3.2) and to determinc the functions $\|_{0}$ and $\|_{1}$ in such a way as to make the new differential equation in $Z$ more closely resemble the given equation. By differentiating $Z$ twice, employing (3.2) at each step to replace $r^{\prime \prime}$ by $R z^{\prime}$, and by constructing the climinant for $z^{\prime}$ and $z^{\prime}$ among the three relations connecting $Z_{,} Z^{\prime}$, and $Z^{\prime \prime}$ with $\tau^{\prime}$ and $\imath^{\prime}$, we find that the differential equation satisfied by functions $\%$ of the form (4.1) is

$$
d^{2 Z}-\begin{gathered}
H \\
d z \\
H_{0} d z
\end{gathered}-\frac{J}{1 \%_{0}} Z=0
$$

wherein

$$
\begin{aligned}
& \mathrm{D}_{0}(z, \lambda)=\left|\begin{array}{cc}
\prime_{0} & \prime_{0}^{\prime}+\mu_{1} R / \lambda \\
\prime_{1} / \lambda & \prime_{0}+\prime_{1}^{\prime} \lambda
\end{array}\right|, \\
& H(\approx, \lambda)=\left|\begin{array}{cc}
\prime_{0} & \prime_{0}^{\prime \prime}+2 \prime_{1}^{\prime} R \lambda_{\lambda}^{1}+\mu_{1} R R^{\prime} \mid \lambda \\
\prime_{1} / \lambda & 2 \prime_{0}^{\prime}+\prime_{1}^{\prime \prime} \mid \lambda
\end{array}\right|,
\end{aligned}
$$

and

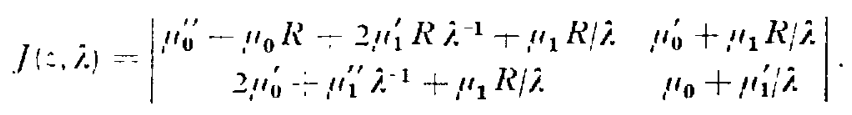

In so far as $\lambda$ is concemed, the functions $\mathrm{D}_{0}$ and $H$ are bounded for $|\lambda|>X^{\circ}$ with $\mathrm{D}_{0}(\approx, \infty)=-\mu^{\prime 2}-\mu^{2} q_{0}$.

We shall determine " ${ }_{0}$ and $"{ }_{1}$ by the condition that the terms in the coe.ficient $J / \mathrm{D}_{0}$ in (4.2) which become infinite with $\lambda$ have sum $\lambda^{2} q_{0}(z)+\lambda q_{1}(z)$. W' recall that the choice of the constant $c$ in (3.2) is in our hands. Now, a simple d.rision 
shows that

$$
\frac{J}{\mathrm{D}_{0}}=R+\mathrm{D}_{0}^{-1}\left|\begin{array}{cc}
\mu_{0}^{\prime \prime}+2 \mu_{1}^{\prime} R \lambda^{-1}+\mu_{1} R^{\prime} \mid \lambda & \mu_{0}^{\prime}+\mu_{1} R / \lambda \\
2 \mu_{0}^{\prime}+\mu_{1}^{\prime \prime} / \lambda & \mu_{0}+\mu_{1}^{\prime} / \lambda
\end{array}\right| .
$$

Recalling the definition of $R$ from (3.2), we observe that the coefficient of $\lambda^{2}$ in $J / \mathrm{D}_{0}$ is $q_{0}$. The coefficient of $\lambda$ may be computed and is

or

$$
c+\mathrm{D}_{0}^{-1}(z, \infty)\left|\begin{array}{cc}
2 \mu_{1}^{\prime} q_{0}+\mu_{1} q_{0}^{\prime} & \mu_{1} q_{0} \\
2 \mu_{0}^{\prime} & \mu_{0}
\end{array}\right|
$$

$$
\mathrm{D}_{0}^{-1}(z, \infty)\left[\left(c \mu_{0}^{2}+2 \mu_{0} \mu_{1}^{\prime} q_{0}+\mu_{0} \mu_{1} q_{0}^{\prime}\right)-\left(c \mu_{1}^{2} q_{0}+2 \mu_{0}^{\prime} \mu_{1} q_{0}\right)\right] \text {. }
$$

Therefore, a sufficient condition that this coefficient be equal to $q_{1}$ is that $\mu_{0}$ and $\mu_{1}$ satisfy the following system of differential equations:

with boundary condition that

$$
\begin{aligned}
2 \mu_{1}^{\prime} q_{0}+\mu_{1} q_{0}^{\prime} & =\mu_{0}\left(q_{1}-c\right), \\
2 \mu_{0}^{\prime} & =\mu_{1}\left(q_{1}-c\right),
\end{aligned}
$$

$$
\mu_{0}^{2}-\mu_{1}^{2} q_{0}=\mathrm{D}_{0}(z, \infty)=1 .
$$

A possible solution of this system is

$$
\mu_{0}=\cosh \vartheta, \quad \mu_{1}=\frac{\sinh \vartheta}{\varphi},
$$

where

$$
\varphi(z)=\left(z^{2}-1\right)^{2}, \quad \arg \varphi=0 \text { for } z>1
$$

and

$$
\vartheta(z)=\int_{1}^{s} \frac{q_{1}(s)-c}{2 \varphi(s)} d s .
$$

We deduce from these formulas that $\mu_{0}$ and $\mu_{1}$ are analytic in $\mathscr{D}_{g}$ except at $z=-1$ provided $\mu_{1}$ is defined so as to be continuous at $z=1$. Without proper choice of the constant $c, \mu_{0}$ and $\mu_{1}$ will fail to be analytic at -1 . In order to render them analytic there, we choose $c$ so that

$$
c=\frac{\int_{-1}^{+1} \frac{q_{1}(s)}{\varphi(s)} d s}{\int_{-1}^{+1} \frac{d s}{\varphi(s)}} .
$$

Then $\vartheta(-1)=0$, and $\vartheta$ may be written in the form

$$
\mathfrak{V}(z)=\int_{-1}^{s} \frac{q_{1}(s)-c}{2 \varphi(s)} d s .
$$

Provided $\mu_{1}$ is now defined so as to be continuous at -1 , this fact and computation show that $\mu_{4}$ and $\|_{1}$ are anilvtic throughout $\mathscr{Q}_{z}$. 
The choice of $\mu_{0}$ and $\mu_{1}$ determine $D_{0}$, and from (4.5) it follows that

$$
\mu_{0}^{2}-\mu_{1}^{2} q_{0}=1 \text {. }
$$

The relations (4.7) and (4.3) and the definition (3.2) of $R$ imply that

$$
\mathrm{D}_{0}(z, \lambda)=1+\lambda^{-1}\left(\mu_{0} \mu_{1}^{\prime}-\mu_{0}^{\prime} \mu_{1}-c \mu_{1}^{2}\right)-\mu_{1}^{2} \sum_{0}^{n} \epsilon_{j} \lambda^{-i-2} \text {. }
$$

Thus if $\mathscr{D}_{z}$ is bounded, it is clear from (4.8) that $D_{0}$ is bounded away from zero for $|\lambda|>N$ and for $z \in \mathscr{D}_{z}$. If $\mathscr{D}_{z}$ is unbounded, we assume

(iii) $\mathrm{D}_{0}$ is bounded away from zero for $z \in \mathscr{D}_{z}$ and for $|\lambda|>N$.

We observe directly from (4.3) that $H=\mathrm{D}_{0}^{\prime}$. Therefore, we may make the change of variable

$$
\zeta=\mathrm{D}_{0}^{-1} Z
$$

to remove the first derivative term in equation (4.2). It then takes the form

$$
\frac{d^{2} \zeta}{d z^{2}}-\left[\lambda^{2}\left(z^{2}-1\right)+\lambda q_{1}(z)+T(z, \lambda)\right] \zeta=0 .
$$

where

$$
\lambda^{2}\left(z^{2}-1\right)+\lambda q_{1}+T=\frac{J}{\mathrm{D}_{0}}+\frac{3}{4}\left(\frac{\mathrm{D}_{0}^{\prime}}{\mathrm{D}_{0}}\right)^{2}-\frac{\mathrm{D}_{0}^{\prime \prime}}{2 \mathrm{D}_{0}^{\prime}} .
$$

The function $T$ is analytic in $\mathscr{D}_{z}$ as $R, \mu_{0}$, and $\mu_{1}$ are all analytic there. Further, $T$ is analytic in $\lambda$ for $|\lambda|>N$; hence, we may write

$$
T(z, \lambda)=\sum_{0}^{\infty} t_{j}(z) \lambda^{-j}
$$

We call the differential equation (4.10a) "the second approximating equation" for the given equation (2.5). It is important to note the way the constants $c_{,}$, which were introduced in (3.1), enter into the functions $t_{j}$. The $c_{j}$ appear in $T$ wherever $R$ and $R^{\prime}$ do. From (4.4) and (4.3a), we observe that aside from the leading term $R$ in the right member of $(4.10 \mathrm{~b}), R$ and $R^{\prime}$ have coefficients of order $\lambda^{-1}$ or smaller in this right member. It follows that the constant $c_{i}$ occurs linearly in $t_{i}$, always with a coefficient of +1 , and is absent from every $t_{j}$ with $j<i$.

5. The related equation. We are now in a position to construct a differential equation whose solutions are known and whose coefficients are identical with those of the given equation up to terms of order $\lambda^{-n-1}$. Inasmuch as this construction is already well known $[5,6]$, we present only the results.

With $\zeta$ standing for any solution of equation (4.10a), we set

$$
w=\mathrm{D}_{1}^{-1}\left(A \zeta+\frac{B \zeta^{\prime}}{\lambda^{2}}\right)
$$

where

$$
\begin{aligned}
& A(z, \lambda)=\sum_{0}^{n} a_{j}(z) \lambda^{-i}, \\
& B(z, \lambda)=\sum_{0}^{n} b_{j}(z) \lambda^{-i},
\end{aligned}
$$


and

$$
\mathrm{D}_{\mathbf{1}}(z, \lambda)=\left|\begin{array}{cc}
A & A^{\prime}+\frac{B S}{\lambda^{2}} \\
\frac{B}{\lambda^{2}} & A+\frac{B^{\prime}}{\lambda^{2}}
\end{array}\right|,
$$

and choose the functions $a_{j}$ and $b_{j}$ so that $w$ satisfies a differential equation of the form

$$
\frac{d^{2} w}{d z^{2}}-\left[\lambda^{2} Q(z, \lambda)-\frac{\Omega(z, \lambda)}{\lambda^{n+1}}\right] w=0,
$$

where $\Omega(z, \lambda)$ is bounded for $z \in \mathscr{D}$ and for $|\lambda|>N$. The proper choices of the $a$,'s and $b_{j}$ 's are:

$$
\begin{aligned}
& a_{0}(z)=1, \quad b_{0}(z)=\frac{1}{\varphi(z)} \int_{1}^{z} \frac{q_{2}(s)-t_{0}(s)}{2 \varphi(s)} d s, \\
& a_{1}(z)=0, \quad b_{1}(z)=\frac{1}{\varphi(z)} \int_{1}^{z}\left[\left(q_{3}-t_{1}\right)-2 q_{1} b_{0}^{\prime}-q_{1}^{\prime} b_{0}\right] \frac{d s}{2 \varphi(s)},
\end{aligned}
$$

(5.3) and for $j=2, \ldots, n$,

$$
\begin{gathered}
a_{i}(z)=\frac{1}{2} \int_{i}^{z}\left[b_{j-2}^{\prime \prime}+\sum_{0}^{j-2}\left(q_{j-1}-t_{j-k-2}\right) b_{k}\right] d s, \\
b_{i}(z)=\frac{1}{\varphi_{i}} \int_{i}^{z_{0}}\left[\sum_{0}^{i}\left(q_{i-k+2}-t_{j-k}\right) a_{k}-\left(2 q_{1} b_{j-1}^{\prime}+q_{1}^{\prime} b_{j-1}\right)-\right. \\
\left.\quad-\sum_{0}^{j-2}\left(2 t_{j-k-2} b_{k}^{\prime}+t_{j-k-2} b_{k}\right)-a_{j}^{\prime \prime}\right] \frac{d s}{2 \varphi} .
\end{gathered}
$$

In order to render the $a$,'s and $b_{j}$ 's analytic in $\mathscr{D}_{z}$, the constants $c_{j}$ occurring in their defining expressions must be properly chosen. This may be done recursively since $c_{i}$ occurs linearly in $t_{i}$ and is absent from each $t_{i}$ with $j<i$. The correct determinations are

$$
c_{0}=\gamma \int_{-1}^{1}\left(q_{2}-t_{0}^{*}\right) \frac{d s}{2 \varphi}, \quad c_{1}=\gamma \int_{-1}^{1}\left[q_{3}-t_{1}^{*}-2 q_{1} b_{0}^{\prime}-q_{1}^{\prime} b_{0}\right]_{2 \varphi}^{d s},
$$

(5.4) and for $j \geqq 2$

wherc

$$
\begin{aligned}
c_{j}=\gamma \int_{-1}^{1}\left[\sum_{1}^{i}\left(q_{j-k}{ }_{2}-t_{j-k}\right) a_{l:}\right. & -\left(2 q_{1} b_{j-1}^{\prime}+q_{1}^{\prime} b_{j-1}\right)+\left(q_{j+2}-t_{i}^{*}\right)- \\
& \left.-\sum_{0}^{1-2}\left(2 t_{j-k-2} b_{k}^{\prime}+t_{j-k-2} b_{k}\right)-a_{j}^{\prime \prime}\right]_{2 \varphi}^{d s},
\end{aligned}
$$

$$
t_{i}^{*}==t_{1}-c_{j} \text { and } \gamma=\left(\int_{-1}^{1} d s\right)^{-1} .
$$

We now observe that the division by $\mathrm{D}_{1}^{1}$ in $\left(5.1\right.$ a) is legitimate if $\mathscr{D}_{z}$ is bounded, since $\mathrm{D}_{1}(z, \infty)=1$. If $\mathscr{D}$ is unbounded, we assume 
(iv) $\mathrm{D}_{1}(z, \lambda)$ is bounded away from zero for $z \in \mathscr{D}_{z}$ and for $|\lambda|>N$.

The function $\Omega(z, \lambda)$ which appears in equation (5.2) is a function whose specific form may be computed, although it is of no interest to us. It involves the several functions $A, B, Q, R, q_{0}$, and $q_{1}$ : It is, however, important to note that $\Omega(z, \lambda)$ is analytic in $\lambda$ for $|\lambda|>N$ and is analytic in $z$ for $z \in \mathscr{D}_{x}$. We henceforward refer to the differential equation (5.2) as the related equation. In Part II we shall single out certain of its solutions and describe their behavior for $z \in \mathscr{D}_{x}$ and $|\lambda|>N$.

\section{Part II: Solutions of the Related Equation}

6. Introduction. The construction just given of the related equation enables one to know the behavior of its solutions only as, well as one knows the behavior of the Weber functions which are solutions of the first approximating equation (3.2). These are Weber functions of large complex order and unrestricted argument, e.g., $D_{1(v-1)}(\sqrt{2 \lambda} z)$. Their behavior is not yet completely known. ERDÉLYI, KenNedy \& MCGREGor have derived the results most useful to us [3]. They have established approximations to the various functions

$$
y_{m}(x, v)=D_{(\sigma v-1) / 2}\left(\sqrt{2 v} e^{\frac{1}{2} m \pi^{i}} x\right), \quad \sigma=(-1)^{m},
$$

with an error involving $\nu^{-1}$. We strengthen their results by showing that their asymptotic representations hold uniformly in arg $v$ over sectors of the $v$-plane. We also extend their representations to asymptotic expansions, the terms of which are determined recursively by a quadrature at each step.

We obtain the behavior of solutions of equation (3.2) by letting $x=\sqrt{\frac{\lambda}{\nu}} z$ in the expansions for the functions (6.1). We then determine the asymptotic expansions over $\mathscr{D}_{z}$ of the solutions of the related equation. These are explicit up to terms involving $v^{-1}$. While our results are limited. by the lack of precise information on the structure of Weber functions of large complex order, approximations to terms involving $\nu^{-1}$ are usually adequate in applications. When they are not, numerical methods for the calculation of the terms of the asymptotic expansions given in $\$ 8$ may be employed to give more precise approximations.

7. Domains in the $\boldsymbol{x}$-plane. The determination of the regions of validity for the approximations to solutions of the given equation, which are derived in Part III, depends upon the character of a function $\Phi(x)$. It is defined by analytic continuation from its positive values on $(1, \infty)$, which are given by

$$
\Phi(x)=\int_{1}^{x}\left(t^{2}-1\right)^{\frac{1}{2}} d t=\int_{1}^{x} \varphi(t) d t .
$$

It is shown in $[3$, pp. 469,470$]$ that $\Phi$ is a schlicht mapping of a Riemann surface $\mathfrak{X}$ over the $x$-plane with branch points at \pm 1 onto a Riemann surface 3 over the $\Phi$-plane whose branch points are the images of the branch points in $\mathfrak{X}$.

Clearly,

$$
\Phi(x)=\frac{1}{2}\left\{x\left(x^{2}-1\right)^{\frac{1}{2}}-\ln \left[x+\left(x^{2}-1\right)^{1}\right]\right\}
$$

and for large $|x|$,

$$
\Phi(x)= \pm \frac{1}{2} x^{2}\left[1+O\left(x^{-1}\right)\right]
$$


The Weber functions (6.1) are entire functions of their argument and their order. Hence, in discussing them we may assume that

$$
\text { The } x \text {-plane is cut from }-1 \text { to }+1 \text { and }|\arg v| \leqq \pi+\varepsilon, \varepsilon>0 \text {. }
$$

These assumptions enable us to avoid considering more than a finite number of sheets of the logarithmic Riemann surface 3 .

Let $\mathfrak{X}_{1}$ denote the $x$-plane cut in accordance with (7.4) and the condition $|\arg (\bar{x}-1)| \leqq \pi$. The corresponding portion $3_{1}$ of 8 consists of two sheets joined
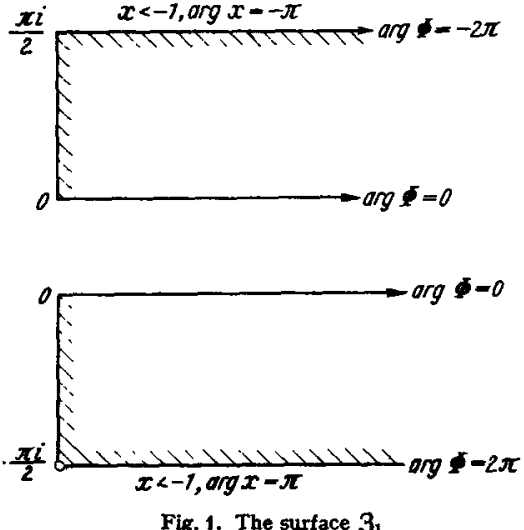
along the ray $\arg \Phi=0$ (see Fig. 1). We now define certain regions of $\mathfrak{X}$ on which the behavior of the Weber functions (6.1) is conveniently describable. Let the index $m$, which appears in (6.1), have range $0, \pm 1, \pm 2, \pm 3$, and let

$$
\xi(x, \nu)=\nu \Phi(x) .
$$

Let $\gamma_{m}$ be the point at infinity on 3 in the direction $\arg \Phi=-m \pi-\arg \nu$, and let $\varepsilon$ be any positive number small enough to make the following definition meaningful. We define $\left(S_{m}\right.$ to be the largest closed, simply connected region $\mathbb{B}$ of $\mathfrak{X}$ such that

(7.6a) $-\frac{3}{2} \pi+\varepsilon \leqq \arg \xi+m \pi \leqq \frac{3}{2} \pi-\varepsilon, \quad x \in \mathbb{S}$,

(7.6b) $|x+1| \geqq \varepsilon, \quad x \in(5)$

(7.6c) $|\Phi(x)| \geqq \varepsilon, x \in(B)$, except that $x=1$ and a neighborhood of $x=1$ may be in $(A)$.

(7.6d) Each point $x$ in \& may be joined to the image of $\gamma_{m}$ by an analytic, simple curve lying in $\mathscr{B}$ and on which $\Re \xi$ is monotone (monotone increasing from the image of $\gamma_{m}$ to $x$ if $m$ is odd, monotone decreasing if $m$ is even).

The regions $\mathbb{S}_{m}$ are illustrated in [3, p. 482]. In general, except for sectors with vertices at $x= \pm 1$ and widths $2 \varepsilon$ in $\arg (x \pm 1)$ and the interior of the circle at $x=-1$ deleted by hypothesis $(7.6 \mathrm{~b})$, each region $B_{m}$ covers the $x$-plane. Exception occurs, for example, when $m=0$ and $\arg \nu=0$.

We denote by $\mathscr{S}_{m}^{*}$ the reflection of $\mathscr{B}_{m}$ in the origin; that is,

$$
\mathscr{B}_{m}^{*}=\left\{x \mid e^{-\pi i} x \in \mathbb{G}_{m} \quad \text { if } \quad \arg x \geqq 0, \quad e^{\pi i} x \in \mathbb{G}_{m} \quad \text { if } \quad \arg x \leqq 0\right\} .
$$

Now

$$
\Phi\left(e^{ \pm \pi i} x\right)=\Phi(x) \mp \frac{1}{2} \pi i,
$$

so that $\mathbb{G}_{m+2}^{*} \cup \mathbb{B}_{m}$ is precisely $\mathscr{B}_{m}$ plus all but a small sector with vertex at $x=-1$ and width $2 \varepsilon$ in $\arg (x-1)$ of the neighborhood of $x=-1$ not in $\mathbb{B}_{m}$. The definitions of $\mathscr{H}_{m}$ and $\mathscr{G}_{m}^{*}$ are essentially the same as those given in [3]. 
8. Asymptotic expansions of Weber functions. In this section we describe the asymptotic behavior of the Weber functions (6.1) over the regions $\mathbb{G}_{m}$ and $\mathbb{B S}_{m}^{*}$. These functions are solutions of the transformed Weber equation

$$
\frac{d^{2} y}{d x^{2}}-v^{2}\left(x^{2}-1\right) y=0 .
$$

Since they are entire functions of $x ; y_{m}=y_{l}$ if $m \equiv l(\bmod 4)$. The Wronskians of certain pairs of these solutions are $[1, \mathrm{p} .42]^{4}$

$$
\begin{aligned}
& \mathscr{W}\left(y_{m}, y_{m \pm 2}, x\right)=\frac{2 \sqrt{\pi v} e^{\frac{1}{3} m \pi i}}{\Gamma\left(\frac{-\sigma v+1}{2}\right)}, \\
& \mathscr{W}\left(y_{m}, y_{m \pm 1}, x\right)=e^{\mp \mathrm{f} \pi i(1+\sigma \nu)} \sqrt{2 v} e^{\frac{1}{3} m \pi i},
\end{aligned}
$$

where

$$
\mathscr{W}(f, g, x)=t \frac{d g}{d x}-g \frac{d f}{d x}
$$

and

$$
\sigma=(-1)^{m}
$$

We henceforth adopt the convention that in formulas in which the double signs \pm or $\mp$ appear, all upper signs or all lower signs are always to be used. It follows from (8.2) that any two of the four distinct solutions $y_{m}$ are linearly independent except for $\nu=0$ and certain other integral values of $y$. If $\nu$ is not zero, and we always consider $|\nu|$ to be large, the solutions $y_{m}$ and $y_{m \pm 1}$ are linearly independent without exception.

The asymptotic representations for the functions $y_{m}(x, \nu)$ involve the Airy function $\mathrm{Ai}(t)$, which is defined by the relation

$$
\operatorname{Ai}(t)=\frac{\sqrt{t}}{\pi \sqrt{3}} K_{1}\left(\frac{2}{3} t^{n}\right),
$$

in which $K_{\mathrm{t}}$ is a modified Bessel function of the third kind [3, p. 463]. For each admissible $m$, the function

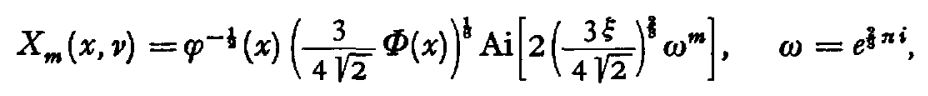

has a simple asymptotic expansion for $x \in \mathbb{G}_{m}$. This is

$$
\begin{aligned}
& X_{m}(x, \nu)=2^{-\frac{1}{2}} \pi^{-\frac{1}{2}} \nu^{-\frac{\xi}{6}} \varphi^{-\frac{1}{2}}(x) e^{-\sigma \xi-m \pi i / 6} \sum_{0}^{N} \frac{\Gamma\left(r+\frac{\delta}{8}\right) \omega^{-3 r m / 2}}{\Gamma\left(-r+\frac{\delta}{8}\right) \nu !(2 \xi)^{r}}+O\left(\xi^{-N-1}\right), \\
& x \in \mathbb{S}_{m}, \quad m=0, \pm 1, \pm 2, \pm 3 .
\end{aligned}
$$

The asymptotic series for $d X_{m} / d x$ for $x \in \mathbb{S}_{m}$ may be obtained from (8.5) by differentiation. ERDÉLYI, KENNFDY \& MCGREGOR have shown that the functions (8.4) are asymptotic representations for the Weber functions (6.1) [3, p. 479]. These representations may be extended to asymptotic expansions by using the algorithm given by LANGER in [5] or similar algorithms. We use LANGER's. In the notation of [5], it enables us to construct certain functions $\alpha_{j}$ and $\beta_{j}$

4 The formulas in [1] appear to contain misprints. 
which are involved in the coefficients of the asymptotic expansions for the Weber functions (6.1). With reference to equation (8.1) the defining expressions of $\alpha_{j}$ and $\beta_{j}$ specialize. They become:

$$
\begin{array}{ll}
\alpha_{0}(x)=1, & \beta_{0}(x)=\frac{1}{\varphi(x)} \int_{i}^{x} \frac{k(t)}{2 \varphi(t)} d t, \\
\alpha_{1}(x)=0, & \beta_{1}(x)=0,
\end{array}
$$

and for $j \geqq 2$,

$$
\begin{aligned}
& \alpha_{j}(x)=\frac{-1}{2} \int_{1}^{r}\left[\beta_{i-2}^{\prime \prime}(t)+k(t) \beta_{j-2}(t)\right] d t \\
& \beta_{j}(x)=\frac{1}{\varphi(x)} \int_{i}^{r}\left\{\alpha_{j}^{\prime \prime}(t)+k(t)\left[\alpha_{j}(t)+2 \beta_{j-2}(t)\right]-k^{\prime}(t) \beta_{j-2}(t)\right\} \frac{d t}{2 \varphi(t)}
\end{aligned}
$$

wherein

$$
k(x)=\frac{\Psi^{\prime \prime \prime}(x)}{\Psi(x)} \quad \text { and } \quad \Psi(x)=\Phi^{1}(x) \varphi^{-1}(x) .
$$

Since $\alpha_{1}=\beta_{1}=0, \alpha_{2 j+1}=\beta_{2 j+1}=0$ for all $j \geqq 0$. The following order relations are easily established. They are important in the extension of the asymptotic representations in [3] to asymptotic expansions. For $|x|>N$ and $j \geqq 0$,

$$
\alpha_{2 j}(x)=O(1), \quad \text { and } \quad \beta_{2 i}(x)=O\left(x^{-1}\right) .
$$

The asymptotic expansions given below extend the results in [3]. We omit their derivation to avoid repetition of work in [3]. The $\alpha_{i}^{\prime}$ s and $\beta_{j}$ 's are sufficiently small at $x=\infty$ to guarantee uniform convergence of the integrals which enter into the derivation. This is the point in the extension of the analysis in [3] that requires careful attention. The bounds for the error terms in our expansions are uniform with respect to $\arg \nu$, if $\arg \nu$ lies on a bounded interval. This also applies to the results in [3]. The reason for this is that the limit $a_{m}$ of integration in [3, equation (4.8)] may be fixed provided arg $v$ has a range of length less than $\pi$. For example, if $|\arg \nu| \leqq \frac{1}{2} \pi-\varepsilon, a_{0}$ may be chosen as $x=e^{0 i} \cdot \infty$; and if $\left|\arg v-\frac{1}{2} \pi\right| \leqq \frac{1}{2} \pi-\varepsilon, a_{0}$ may be chosen as $x=e^{-1 \pi i} \cdot \infty$. For each such range of arg $\nu$, a bound on the integral in [3, equation (4.8)] may be found which is independent of arg $\nu$. It follows that for $\arg \nu$ bounded, bounds for the error terms independent of arg $v$ can be found. This reasoning does not imply, however, that in the $x$-plane the regions of validity for the asymptotic expansions below are independent of $\arg v$. They are not, although in the neighborhood of infinity the boundaries of the regions of validity in the $\sqrt{\nu x}$ plane are asymptotically independent of $\arg v$.

for $x \in \mathfrak{G}_{m}$; and

$$
\begin{aligned}
y_{m}(x, v)= & C_{m}\left[D_{1}^{*}(x, v)\right]^{-1} \times \\
& \times\left\{A^{*}(x, v) X_{m}(x, v)\left[1+O\left(v^{-j-1}\right)\right]+\frac{B^{*}(x, v)}{v^{2}} \frac{d X_{m}(x, v)}{d x}\right\},
\end{aligned}
$$

$$
\begin{aligned}
y_{m}(x, v)= & C_{m}\left[D_{1}^{*}(x, v)\right]^{-\frac{1}{2}} \times \\
& \times\left\{A^{*}(x, v) X_{m}(x, \nu)+\frac{B^{*}(x, \nu)}{\nu^{2}} \frac{z X_{m}(x, v)}{d x}+O\left(\nu^{-j-1}\right)\right\},
\end{aligned}
$$


for all $x \in\{x|| x-1 \mid<\varepsilon\}$ and $|\xi(x, \nu)|<N$. In these relations

$$
\begin{aligned}
& C_{m}=2 \pi^{\frac{1}{2} \nu^{1\left(\sigma \nu-\frac{1}{3}\right)}} e^{-\frac{1}{1}\left[\sigma \nu(1-m \pi i)+\frac{1}{3} m \pi i\right]}, \\
& D_{1}^{*}(x, y) \\
& =\left|\begin{array}{ll}
A^{*}(x, v) & \nu^{-2} B^{*}(x, v) \\
A^{* \prime}(x, v)-\left(x^{2}-1\right) B^{*}(x, v)+k(x) B^{*}(x, v) \nu^{-2} & A^{*}(x, v)+\nu^{-2} B^{* \prime}(x, v)
\end{array}\right|,
\end{aligned}
$$

and

$$
A^{*}(x, \nu)=\sum_{0}^{i-1} \alpha_{i}(x) \nu^{-i}, \quad B^{*}(x, \nu)=\sum_{0}^{j-1} \beta_{i}(x) \nu^{-i}
$$

The functions $\alpha_{i}, \beta_{i}$, and $k$ are defined by the relations (8.6), the functions $X_{m}(x, \nu)$ are described by the formulas (8.4) and (8.5), and $\sigma=(-1)^{m}$.

One uses the identity

$$
y_{m}(x, v)=y_{m \pm 2}\left(e^{\mp z i} x, v\right)
$$

to derive the form of $y_{m}(x, v)$ when $x \in \mathscr{G}_{m=2}^{*}$. Taken together the regions $\mathbb{S}_{m}$ and $0_{m}^{*}(m=0, \pm 1, \pm 2, \pm 3)$ cover the $x$-plane, each point being in two regions having indices which differ by 1 or 3 . Therefore, the expansions (8.8) yield the asymptotic behavior of two linearly independent solutions of the differential equation (8.1) for each $x$. We observe that zeros of the Airy function are not excluded from the region where the relation $(8.8 \mathrm{~b})$ is fulfilled. This is the reason for the altered character of the error term in $(8.8 \mathrm{~b})$ as compared with the error term in [3, equation (9.6)], for example. The behavior of $d y_{m} / d x$ may be found via the relation

$$
\frac{d D_{\nu}(x)}{d x}=\nu D_{v-1}(x)-\frac{1}{2} x D_{v}(x)
$$

from the expansions (8.8).

It is interesting to note that if $v$ is real, all solutions of equation (8.1) are oscillatory on the interval $-1<x<1$. This occurs since, for the configuration of $x$ and $\nu$ cited, $\Re(\xi(x, v))=0$.

9. Solutions of the related equation. The first approximating equation (3.2) has solutions

$$
v_{m}^{\prime}(z, \lambda)=D_{1(\sigma p-1)}\left(l^{\prime} 2 \lambda e^{\left.\frac{1}{m \pi i} z\right)} \quad(m=0, \pm 1, \pm 2, \pm 3) .\right.
$$

where $D_{\nu}(z)$ is the standard parabolic cylinder function of order $\nu[1]$, and where

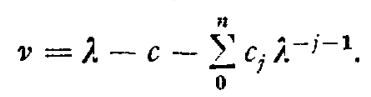

Of course, $v_{m}=v_{l}$ if $l \equiv m(\bmod 4)$. The formula

$$
v_{m}(z, \lambda)=y_{m}\left(\left.\right|_{y} ^{i} z, v\right)
$$

relates the solutions of equations (3.2) and (8.1). In view of this connection, the asymptotic behavior of the solutions $y_{m}\left(z, \lambda_{i}\right)$ may now be easily described with reference to the $x$-plane. Since

$$
x=\prod_{i}^{i},
$$

the transition from regions on the $x$-plane to their images on the $z$-plane is elementary. 
By directly applying the theory in [5] to equation (3.2), we could have described the behavior of certain solutions of (3.2), other than the solutions (9.1), on regions in the $z$-plane identical with the regions $\mathscr{G}_{m}$ and $\mathscr{G}_{m}^{*}$. However, the slight simplification in the description of the regions of validity of the asymptotic expansions, which is gained by this approach, is offset by an increase in difficulty in deriving these expansions, since equation (3.2) is superficially more complicated than equation (8.1). For this reason and to take fullest advantage of the work in [3], we proceeded as we have done.

We compute the Wronskians of the solutions $v_{m}(z, \lambda)$ using the formulas (8.2), (9.3), and (9.4). For future reference, the computed values are:

$$
\begin{aligned}
& \mathscr{W}\left(v_{m}, v_{m \pm 2}, z\right)=\frac{2 l \pi \lambda e^{\frac{1}{2} m \pi i}}{\Gamma\left(\frac{-\sigma v+1}{2}\right)}, \\
& \mathscr{W}\left(v_{m}, v_{m \pm 1}, z\right)=e^{\mp i \pi i(1+\sigma v)} \sqrt{2 \lambda} e^{\frac{1}{2} m \pi i} .
\end{aligned}
$$

To each solution $v_{m}(z, \lambda)$ of equation (3.2) there corresponds, through the relations $(4.1),(4.9)$, and $(5.1)$, a solution $w_{m}^{\prime}(z, \lambda)$ of the related equation. The solutions $w_{m}(z, \lambda)$ may be written in terms of the functions $v_{m}(z, \lambda)$ as follows:

$$
w_{m}(z, \lambda)=E_{0}(z, \lambda) v_{m}(z, \lambda)+E_{1}(z, \lambda) v_{m}^{\prime}(z, \lambda) / \lambda,
$$

where

$$
\begin{aligned}
& E_{0}(z, \lambda)=\left(D_{0} D_{1}\right)^{-\frac{1}{2}}\left[A \mu_{0}+\frac{B}{\lambda}\left(\frac{\mu_{1} R}{\lambda^{2}}+\frac{\mu_{0}^{\prime}}{\lambda}-\frac{\mu_{0} D_{0}^{\prime}}{2 \lambda D_{0}}\right)\right], \\
& E_{1}(z, \lambda)=\left(D_{0} D_{1}\right)^{-\frac{1}{2}}\left[A \mu_{1}+\frac{B}{\lambda}\left(\mu_{0}+\frac{\mu_{1}^{\prime}}{\lambda}-\frac{\mu_{1} D_{0}^{\prime}}{2 \lambda D_{0}^{\prime}}\right)\right] .
\end{aligned}
$$

Since

$$
\mathscr{W}\left(w_{l}, w_{m}, z\right)=\mathscr{W}\left(v_{l}, v_{m}, z\right),
$$

the linear independence of solutions $w_{l}$ and $w_{m}$ depends upon that of their correspondents $v_{l}$ and $v_{m}$.

For the proofs to follow it is necessary to have at hand the asymptotic behavior, in first approximation, as $|\lambda| \rightarrow \infty$, of the solutions $w_{m}(z, \lambda)$ of the related equation together with the behavior of their first derivatives. One finds this behavior by considering the relations (9.6) in conjunction with the asymptotic expansions (8.8) and (8.5) and the relation (8.4). When $|\xi(x, \nu)| \leqq M$ and $|x-1|<\varepsilon$

$$
\varkappa_{m}^{\prime}(z, \lambda)=C_{m} O(1), \text { and } w_{m}^{\prime}(z, \lambda)=C_{m} O\left(\lambda^{i}\right) \text {. }
$$

When $|\xi(-x, v)| \leqq M$ and $|x+1|<\varepsilon$,

$$
w_{m}(z, \lambda)=C_{m \pm 2} O(1), \text { and } w_{m}^{\prime}(\tilde{z}, \lambda)=C_{m \pm 2} O\left(\lambda^{\tilde{3}}\right) \text {. }
$$

If $z$ is bounded, $x \in \mathcal{G}_{m} \cup\left(\mathcal{S}_{m \pm 2}^{*},|\xi(x, v)|>M\right.$, and $|\xi(-x, v)|>M$, then

$$
\begin{aligned}
& w_{m}^{\prime}(z, \lambda)=\Lambda_{m}(z, \lambda) O\left(\lambda^{-1}\right), \\
& w_{m}^{\prime}(z, \lambda)=w_{m}^{\prime}(z, \lambda) O(\lambda),
\end{aligned}
$$

where

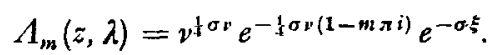


If $z$ is not bounded, then the functions $E_{0}$ and $E_{1}$ defined by (9.7) may be unbounded. In this case, the relations $(9.10 \mathrm{a})$ and $(9.10 \mathrm{~b})$ are invalid. We therefore introduce a function $b(z)$ which is continuous, which is nonvanishing for $|z|>M$ and equal to 1 for $|z| \leqq M$, and which is the "largest" function such that for each $m$ (9.11) $\left|b(z) \Lambda_{m}^{-1}(z, \lambda) w_{m}(z, \lambda)\right|<N|v|^{-1}$ and $\left|b(z) \Lambda_{m}^{-1}(z, \lambda) \frac{w_{m}^{\prime}(z, \lambda)}{\lambda}\right|<N|v|^{-1}$, when $x \in \mathscr{S}_{m} \cup \mathbb{S}_{m \pm 2}^{*}$ and when $|\xi(x, \nu)|>M$ and $|\xi(-x, v)|>M$. Thus when the relations (9.11) apply,

and

$$
w_{m}(z, \lambda)=\frac{\Lambda_{m}(z, \lambda) O\left(\lambda^{-1}\right)}{b(z)}
$$

$$
{ }^{\prime} e_{m}^{\prime}(z, \lambda)=\frac{A_{m}(z, \lambda) O\left(\lambda^{2}\right)}{b(z)}
$$

\section{Part III: Asymptotic Expansions for Solutions of the Given Equation}

10. Final hypotheses on $\mathscr{D}_{z}$. Our objective is to determine the asymptotic behavior throughout $\mathscr{D}_{z}$ of a pair of linearly independent solutions of the given equation (2.5). This will be done in the sections to follow. We first link the behavior of solutions of the given equation to that of solutions of the related equation by using the familiar method of variation of parameters. Its application to the given equation yields the integral equation

$$
u=w+\int_{z_{a}}^{z} \frac{w_{a}(z) w_{b}(t)-w_{a}(t) w_{b}(z)}{\mathscr{W}\left(w_{a}, w_{b}, t\right)} \frac{\Omega(t, \lambda)}{\lambda^{n+1}} u(t) d t,
$$

which is equivalent to the given equation in the sense that an analytic solution of either one is a solution of the other. In equation (10.1), $w_{a}$ and $w_{3}$ may be any pair of linearly independent solutions of the related equation, $w$ may be any solution of the related equation, and $z_{*}$ may be any point in $\mathscr{D}_{8}$. The kernel in equation (10.1) is, of course, independent of the choice of $w_{a}$ and $w_{b}$ so that a unique solution $u$ of the given equation is determined by specifying $w$ and $z_{*}$ in equation (10.1).

The variables $z$ and $x$ and the parameters $\lambda$ and $\nu$ are always considered to fulfill the relations (9.4) and (9.2). Thus, given $z$ and $\lambda, v$ and $x$ are determined. In order to keep the investigation to a reasonable length and to avoid further notational complications, we make the assumption that

$$
|\arg \lambda| \leqq \pi \text {. }
$$

The second of the conditions (7.4) is thereby fulfilled when $|\lambda|$ is sufficiently large. There is no loss of generality in assuming that the first of these conditions is also satisfied. In what follows we fix $\lambda$, once for all, with $|\lambda|$ sufficiently large to fulfill all requirements placed upon it.

The following lemma will be used in the discussion of solutions of (10.1).

Lemma. If

a) $\mathscr{A}$ is a closed, simply connected region (whose boundary may depend upon a complex parameter $\lambda$ ). 
b) $f(z, \lambda)$ is continuous and bounded for $z \in \mathscr{A}$ and for $|\lambda|>N$,

c) $K(z, t, \lambda)$ is continuous for $z \in \mathscr{A}$, for $t \in \mathscr{A}$, and for $|\lambda|>N$,

d) there exist a constant $M$, a point $s \in \mathscr{A}$, and a set of rectifiable analytic curies oining the points of $\mathscr{A}$ to such that for all $z \in \mathscr{A}$

$$
\int_{s}^{z}|K(z, t, \lambda)||d t|<M,
$$

then the solution of the integral equation

has the form.

$$
g(z, \lambda)=f(z, \lambda)+\lambda^{-k} \int_{s}^{\tilde{z}} K(z, t, \lambda) g(t, \lambda) d t \quad(k>0)
$$

$$
g(z, \lambda)=f(z, \lambda)+\lambda^{-k} O(1) .
$$

where $O(1)$ denotes a function of $z$ and $\lambda$ which is uniformly bounded for $z=\mathscr{A}$ and for $|\lambda|>N$.

The hypotheses of the Lemma guarantee the uniform convergence of the series which is obtained by successive iteration of the integral equation, and from this the proof of the Lemma follows.

For the Lemma to apply to the integral equation (10.1), it is necessary that the kernel in (10.1) be bounded. We now make assumptions on $\mathscr{D}_{z}$ sufficient to establish the boundedness of the kernel. For each $\lambda$, let

$$
\mathscr{R}_{m}=\left\{z \mid x(z) \in \mathcal{B}_{m} \cup \mathbb{B}_{m \leq 2}^{*}\right\} \quad(m=0, \pm 1, \pm 2, \pm 3) .
$$

In this definition and in the sequel, when a region with subscript larger than 3 or less than -3 appears, the region is understood to be the null set. Because of the overlapping of the regions $\left(B_{m}\right.$ and also of the regions $\left(B_{m}^{*}\right.$, one can show that $\mathscr{R}_{m}<\mathscr{R}_{m-1} \cup \mathscr{R}_{n+1}(n=0, \pm 1, \pm 2)$. It follows from the hypothesis (v) and the conditions (7.4) that we lose no generality by assuming that $\mathscr{R}_{-3}<\mathscr{R}_{-2}$ and $\mathscr{R}_{3}<\mathscr{R}_{2}$. The regions $\mathscr{R}_{m}$ cover $\mathscr{D}_{z}$ and each point of $\mathscr{D}_{z}$ is in at least two such regions. Our final assumptions on $\mathscr{D}_{z}$ are:

(vi) Corresponding to each region $\mathscr{R}_{m}$, there is a point $z_{m}$ such that each point $z \subseteq \mathscr{R}_{m}$ may be joined to $z_{m}$ by an analytic curve $I$ lying in $\mathscr{R}_{m}$ and such that on $\Gamma$, $9 i \xi$ is monotone (monotone increasing from $z_{m}$ to $z$ if $m$ is odd, monotone dicreasing from $z_{m}$ to $z$ if $m$ is even).

(vii) The integrals

$$
\int_{i} \frac{Q(t, \lambda) d t}{b^{2}(t)}
$$

are uniformly bounded for $\approx \in \mathscr{Q}_{z}$ and for $|\lambda|>\Lambda$.

The function $\Omega(z, \lambda)$ first appears in equation (5.2). The function $b(t)$ is defined by the conditions (9.11). Of course, if $\mathscr{D}$; is bounded, hypothesis (vii) is automatically fulfilled.

There are certain shadow zones which must be excluded from the regions $\mathscr{R}_{t}$ in the discussion of subdominant solutions of the given equation. Their images on the $x$-plane adjoin the segment $-1<x<0$. Their size and presence depends 
upon $\arg \lambda$. They are defined as follows:

$$
\mathscr{S}_{m}=\left\{z\left|z \in \mathscr{R}_{m}, \Re(\sigma \xi)>N,\right| \xi(-x(z)) \mid>N,\right.
$$

and the paths joining $z$ to $z_{m}$ all pass through

the neighborhood of $\mathscr{R}_{m}$ where $\left.|\xi(-x)|<N\right\}$.

The shadow zones are excluded for the reason that the subdominant solutions of the given equation undergo a change in their asymptotic forms as one crosses into them. The presence of these shadow zones (see Fig. 2 for an example) is a phenomenon which has no counterpart in the discussion of asymptotic solutions of differential equations having just one turning point.

As we have remarked, we wish to determine the asymptotic structure throughout $\mathscr{D}_{z}$ of a pair of linearly independent solutions of the given equation. We first prove that, for each $m$, there exists a solution $u_{m}$ which is asymptotically represented by $w_{m}$ in the region $\mathscr{R}_{i n}-\mathscr{S}_{m}$. We then derive connection formulas

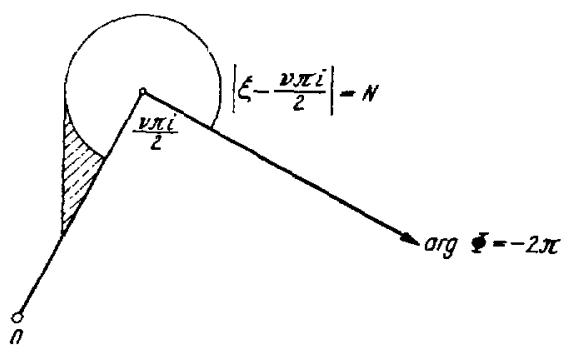

Fig. 2. A shadow zone among the solutions $u_{m}$ from which the behavior of the solutions $u_{m}$ may be derived for all $z \in \mathscr{D}_{z}$. Lastly, we discuss certain characteristic solutions, which may well be of special interest in applications of the theory.

11. Subdominant solutions when $|\xi( \pm x, v)|>N$. For each region $\mathscr{R}_{m}-\mathscr{S}_{m}$ there is a solution of the given equation which is subdominant, exponentially small, for $z \in\left(\mathscr{R}_{m}-\mathscr{S}_{m}\right)$ and $\Re(\sigma \xi)>0$. This solution is unique up to a constant factor. We now establish its existence and give its asymptotic properties. We assume for the moment that $z \in\left(\mathscr{R}_{m}-\mathscr{S}_{m}\right) \cap \mathscr{R}_{m+1}$. In equation (10.1) let $w_{a}^{\prime}-w_{m}$ and $w_{b}=w_{m+1}$, let $w=w_{m}$, and let the path of integration be a curve $\Gamma$ which originates at $z_{m}$. For brevity, we adopt the notation $\tau=\xi(x(t), v)$ and $\xi=\xi(x(z), \nu)$. If the curve $\Gamma$ should intersect one or both of the regions where $|\tau( \pm x)| \leqq N$, then $\Re(\sigma \xi) \leqq 0$. Thus, if we replace the subarcs of $\Gamma$ on which $|\tau( \pm x)| \leqq N$ by arcs on which $|\tau( \pm x)|=N$, and if we note that on the remaining portion of $\Gamma, \Re(\sigma \xi) \leqq \Re(\sigma \tau)$, then we see that on the (new) path of integration $\exp [2 \sigma(\xi-\tau)]$ is bounded.

The choices just made determine a solution $u_{m}$ of equations (10.1) and (2.5). If we adopt the abbreviations

$$
\begin{aligned}
& U_{m}(z)=\Lambda_{m}^{-1} b(z) u_{m}(z, \lambda), \\
& W_{m}(z)=\Lambda_{m}^{-1} b(z) w_{m}(z, \lambda),
\end{aligned}
$$

where $\Lambda_{m}(z, \lambda)$ and $b(z)$ are defined by $(9.10 \mathrm{c})$ and $(9.11)$, we can rewrite the integral equation (10.1) in the form

$$
U_{m}(z)=W_{m}(z)+\int_{\Gamma}\left\{W_{m}(z) W_{m+1}(t)-W_{m}(t) W_{m+1}(z) e^{2 \sigma(\xi-\tau)}\right\} \frac{o(1) \Omega(t) U_{m}(t)}{b^{2}(t) \lambda^{n+2}} d t
$$

In writing (11.2), we have used the evaluations (9.8) and (9.5) of $\mathscr{W}\left(w_{m}, w_{m+1}, z\right)$. The relations (9.10), (9.12) and (11.1) imply that for $|\xi( \pm x, \nu)|>N$ and 
$z \in\left(\mathscr{R}_{m}-\mathscr{S}_{m}\right) \cap \mathscr{R}_{m+1}$, both $W_{m}$ and $W_{m+1}$ are bounded. We have already observed that $\exp [2 \sigma(\xi-\tau)]$ is bounded on $\Gamma$. All functions in equation (11.2) are continuous. In virtue of these facts and hypothesis (vi) of $\S 10$, the hypotheses of the Lemma are fulfilled with respect to equation (11.2). Thus for $z \in\left(\mathscr{R}_{m}-\mathscr{S}_{m}\right) \cap$ $\mathscr{R}_{m+1},|\xi( \pm x(z), v)|>N$, and $|\lambda|>N$,

$$
U_{m}(z)=W_{m}(z)+\lambda^{-n-2} O(1) .
$$

We can show by analysis analogous to that above that this relation also holds for $z \in\left(\mathscr{R}_{m}-\mathscr{S}_{m}\right) \cap \mathscr{R}_{m-1}$. In terms of $u_{m}$ and $w_{m}$, the result (11.3) takes the form

$$
u_{m}(z, \lambda)=w_{m}(z, \lambda)+\frac{A_{m}(z, \lambda) O(1)}{b(z) \lambda^{n+2}}: \quad(|\lambda|>N) .
$$

Since $\left(\mathscr{R}_{m}-\mathscr{S}_{m}\right)=<\mathscr{R}_{m-1} \cup \mathscr{R}_{m+1}$, (11.4) holds for all $z \in\left(\mathscr{R}_{m}-\mathscr{S}_{m}\right)$ such that $|\xi( \pm x(z), v)|>N$.

We can use the estimates (11.4) and (9.12) to obtain the the form of $u_{m}^{\prime}(z, \lambda)$ directly from the equation found by differentiation of (10.1). It is

$$
u_{m}^{\prime}(z, \lambda)=w_{m}^{\prime}(z, \lambda)+\frac{A_{m}(z, \lambda) O(1)}{b(z) \lambda^{n+1}}
$$

where the prime denotes differentiation with respect to $z$.

12. The solutions $\boldsymbol{u}_{m}$ when $|\xi( \pm x, v)| \leqq N$. There are two cases to be considered: $|\xi(x, v)| \leqq N$ and $|\xi(-x, v)| \leqq N$. For the moment we suppose that $|\xi| \leqq N$. We recall that the solution $u_{m}$ of the given equation and equation (10.1) is that solution determined by the choices $w=w_{m}$ and $z_{*}=z_{m}$ in (10.1), the path of integration being a curve $\Gamma$. We divide $\Gamma$ in two parts $\Gamma_{1}$ and $\Gamma_{2}$ on which $|\tau| \geqq N$ and $|\tau|<N$, respectively. As was done in $\S 11$, we replace any part of $\Gamma_{1}$ on which $|\tau(-x)|<N$ by an arc on which $|\tau(-x)|=N$. We now write the integral equation for $u_{m}$ in the form

$$
u_{m}(z)=w_{m}(z)+\int_{\Gamma_{1}} K(z, t, \lambda) u_{m}(t) d t+\int_{\Gamma_{z}} K(z, t, \lambda) u_{m}(t) d t
$$

where

$$
K(z, t ; \lambda)=\frac{w_{m}(z) w_{m \pm 1}(t)-w_{m}(t) w_{m \pm 1}(z)}{\mathscr{W}\left(w_{m}^{\prime}, w_{m \pm 1}^{\prime}, t\right)} \cdot \frac{\Omega(t, \lambda)}{\lambda^{n+1}},
$$

the upper or lower signs being used according as $\Gamma_{1}<\mathscr{R}_{m+1}$ or $\Gamma_{1}<\mathscr{R}_{m-1}$.

For the sake of argument let $\Gamma_{1}<\mathscr{R}_{m+1}$. On $\Gamma_{1}$, the behavior of $u_{m}$ is then described by formula (11.4). Using this result and the relations (9.9a), (9.10), and (9.12), we find that

$$
\begin{aligned}
\int_{\Gamma_{1}} K(z, t, \lambda) u_{m}(t) d t & =C_{m}^{2} C_{m+1} \int_{\Gamma_{1}} \frac{\left[e^{\sigma \tau} O(1)-e^{-\sigma \tau} O(1)\right]}{e^{\mp i \sigma \alpha}} \cdot \frac{\Omega(t, \lambda) e^{-\sigma \tau} O(1)}{b^{2}(t) \lambda^{n+\frac{11}{k}}} d t, \\
& =\frac{C_{m}}{\lambda^{n}+2} \int_{\Gamma_{1}}\left[O(1)+e^{-2 \sigma \tau} O(1)\right] \frac{\Omega(t, \lambda)}{b^{2}(t)} d t .
\end{aligned}
$$

But $\Re(\sigma \tau) \geqq 0$ on $\Gamma_{1}$, except perhaps for an arc on which $\Re(\sigma \tau) \geqq-N$. Therefore by hypothesis ;vii),

$$
\int_{\Gamma_{\mathrm{t}}} K(z, t, \lambda) u_{m}(t, \lambda) d t=\frac{C_{m} O(1)}{\lambda^{n+2}}
$$


From the estimate (9.9a) and the order relation $\int_{\Gamma_{2}} d t=O\left(\lambda^{-\frac{2}{3}}\right)$, we deduce that

$$
\int_{\Gamma_{2}} K(z, t, \lambda) d t=\lambda^{-\left(n+\frac{z}{3}\right)} \int_{\Gamma_{z}} \lambda^{\frac{2}{3}} O(1) d t=\lambda^{-\left(n+\frac{z}{8}\right)} O(1) .
$$

The estimate (12.2) enables us to rewrite equation (12.1) as

$$
\frac{u_{m}}{C_{m}}=\frac{w_{m}}{C_{m}}+\lambda^{-n-2} O(1)+\int_{\Gamma_{2}} K(z, t, \lambda) \frac{u_{m}(t)}{C_{m}} d t .
$$

The relation (12.3) shows that the hypotheses of the Lemma are fulfilled with respect to this equation; hence,

$$
u_{m}(z, \lambda)=w_{m}(z, \lambda)+C_{m} \frac{O(1)}{\lambda^{n+2}} \quad(|\xi(x, v)| \leqq N) .
$$

Since the order relation (9.9a) is independent of $\arg (z-\sqrt{\nu / \lambda})$, this result is valid in a full neighborhood of $x=1$, or of $z=\sqrt{\nu / \lambda}$, where $|\xi| \leqq N$ and not just in that portion of $\mathscr{R}_{m}$ where $|\xi| \leqq N$.

We now consider the case $|\xi(-x)| \leqq N$. This situation is more complicated. If, for such $z, \Re(\sigma \xi)<-N$, then the estimate (12.2) no longer holds; and therefore we cannot determine the behavior of $u_{m}$ when $|\xi(-x)| \leqq N$ as we did above for $|\xi| \leqq N$. We resolve this difficulty by showing that it is only an apparent one. Let us momentarily suppose that $m=\mp 2$ and $\Re(\sigma \xi) \leqq-N$. Then $\Re(\sigma \xi) \geqq N$ in the neighborhood of $\mathscr{R}_{m \pm 4}$ where $|\xi(-x)| \leqq N$. By the argument given in the next paragraph we are able to determine the behavior of $u_{m \pm 4}$ in a full neighborhood of $z=-\sqrt{\nu / \lambda}$, or of $x=-1$, where $|\xi(-x)| \leqq N$. We are also able to determine the behavior of $u_{m \pm 1}$ in such a neighborhood. Similar considerations apply to values of $m$ other than \pm 2 . Thus, we shall always be able to determine the behavior of two linearly independent solutions of the given equation in a full neighborhood of $z=-\sqrt{v / \lambda}$ where $|\xi(-x)| \leqq N$.

As promised above, we now obtain the form of $u_{m}$ if $|\xi(-x)| \leqq N$ and if, for such $z, \Re(\sigma \xi) \geqq-N$. To do this we replace $w_{m}(z)$ and $w_{m \pm 1}(z)$ in (12.1) by $w_{m \pm 2}\left(e^{\mp \pi i} z\right)$ and $w_{m \mp 1}\left(e^{ \pm \pi i} z\right)$, respectively, and similarly replace $w_{m}(t)$ and $w_{m \pm 1}(t)$ for $\tau$ such that $|\tau(-x)|<N$. In virtue of the identity (8.10), the analysis used in the derivation of (12.4) may be easily modified to yield the conclusion that

$$
u_{m}(z, \lambda)=w_{m \pm 2}\left(e^{\mp \pi i} z, \lambda\right)+\frac{C_{m \pm 2} O(1)}{\lambda^{n+2}} .
$$

when $|\xi(-x, \nu)| \leqq N$ provided that for such $z, \Re(\sigma \xi) \geqq-N$. In (12.5) the upper signs are to be used if $m<0$, lower signs if $m>0$, and either if $m=0$. As is the case when $|\xi|<N$, we need not impose the restriction here that $z$ lie in $\mathscr{R}_{m}$. We need only suppose that the portion of the path of integration on which $|\xi(-x)| \leqq N$ is rectifiable. Thus the result (12.5) is valid in a full neighborhood of $z=-\sqrt{\nu / \lambda}$ where $|\xi(-x)| \leqq N$.

We can use the estimates of $u_{m}$ obtained thus far to find the form of $u_{m}^{\prime}(z, \lambda)$ when $|\xi| \leqq N$ or $|\xi(-x)| \leqq N$ directly from the equation obtained by differentiation of (10.1). The results are:

$$
\begin{aligned}
& u_{m}^{\prime}(z, \lambda)=w_{m}^{\prime}(z, \lambda)+\frac{C_{m} O(1)}{\lambda^{n+1}} \\
& (|\xi(x, \nu)| \leqq N), \\
& u_{m}^{\prime}(z, \lambda)=w_{m \pm 2}^{\prime}\left(e^{\mp \pi i} z, \lambda\right)+\frac{C_{m \pm 2} O(1)}{\lambda^{x+1}} \\
& (|\xi(-x, v)| \leqq N) \text {, }
\end{aligned}
$$

in neighborhoods of $z=\sqrt{\nu / \lambda}$ and $z=-\sqrt{v / \lambda}$, respectively. 
We summarize our results in a theorem:

Theorem 1. Under the hypotheses (i) through (vii) and corresponding to each region $\mathscr{R}_{m}$, the given equation (2.5) has a solution $u_{m}$ with the following asymptotic behavior when $|\lambda|>N$ :

a) When $z \in\left(\mathscr{R}_{m}-\mathscr{S}_{m}\right)$ and $|\xi( \pm x, v)|>N, u_{m}$ is exponentially large or small according as $\Re(\sigma \xi)<0$ or $\Re(\sigma \xi)>0$ and is described by the formulas (11.4) and (11.5).

b) When $|z-\sqrt{\nu \mid \lambda}| \leqq \varepsilon$ and $|\xi(x, v)| \leqq N$ or $|z+\sqrt{v / \lambda}| \leqq \varepsilon$ and $|\xi(-x, \nu)| \leqq N$, $u_{m}$ is oscillatory in character and is described by the relations (12.4), (12.6a) and (12.5), (12.6b), respectively, provided $\sqrt{\nu / \lambda}$ and $-\sqrt{\nu / \lambda}$ lie in $\mathscr{R}_{m}$ and $\Re(\sigma \xi) \geqq-N$.

c) If $v$ is real, then $u_{m}$ is bounded and oscillatory for $-1 \leqq x \leqq 1, m=0, \pm 1, \pm 2$.

The regions $\mathscr{R}_{m}$ and $\mathscr{S}_{m}$, the variables $x$ and $\xi$, and the parameters $\nu$ and $\sigma$ are defined by $(10.2),(10.3),(9.4),(7.5),(9.2)$, and (6.1), respectively. The functions and constants appearing in the relations (11.4), (11.5), (12.4), (12.5) and (12.6) are defined in Parts I and II. The bounds for the error terms in all of the estimates for solutions of the given equation which have been derived are uniform in $\arg \lambda$ for $|\arg \lambda| \leqq \pi$. This is so for the same reasons as those cited in $\S 8$ for the uniform boundedness of the error terms in the expansions $(8.8 \mathrm{a})$ and $(8.8 \mathrm{~b})$ with respect to $\arg v$.

13. Connection formulas. It remains to give a description of the solutions $u_{m}(z, \lambda)$ when $|\xi( \pm x)|>N$ and when $z$ is not confined to the specific region $\mathscr{R}_{m}$ as assumed in Theorem 1. The dependence relation among three solutions $u_{l}$, $u_{m}$, and $u_{m+1}$ of the given equation may be written in the form

$$
u_{l}=A_{l, m} u_{m}+B_{l, m} u_{m+1} .
$$

The coefficients are, of course, given by the formulas

$$
A_{l, m}=\frac{\mathscr{W}\left(u_{l}, u_{m+1}, z\right)}{\mathscr{W}\left(u_{m}, u_{m+1}, z\right)}, \quad B_{l, m}=\frac{\mathscr{W}\left(u_{m}, u_{l}, z\right)}{\mathscr{W}\left(u_{m}, u_{m+1}, z\right)}
$$

Since the given equation lacks a first derivative term, the Wronskians in (13.2) are all constants and may be evaluated at $z=\sqrt{\nu / \lambda}$, where $x=1$ and $\xi=0$. If each of the solutions $u_{l}, u_{m}$, and $u_{m+1}$ fulfills the relation (12.4), then we may conclude that

$$
\mathscr{W}\left(u_{k}, u_{j}\right)=\mathscr{W}\left(w_{k}, w_{j}\right)+C_{k} C_{j} O\left(\lambda^{-n-1}\right),
$$

where $k, j=l, m$, or $m+1$. Thus by relation (9.8),

$$
\begin{aligned}
& A_{l, m}=\frac{\mathscr{W}\left(v_{l}, v_{m+1}\right)}{\mathscr{W}\left(v_{m}^{\prime}, v_{m+1}^{\prime}\right)}+C_{m+1} C_{l} e^{\frac{l}{\sigma \pi^{i \nu}}} O\left(\lambda^{-n-3 / 2}\right), \\
& B_{l, m}=\frac{\mathscr{W}\left(v_{m}, l_{l}^{\prime}\right)}{\mathscr{W}\left(v_{m}, v_{m+1}\right)}+C_{m} C_{l} e^{l \sigma \pi r^{i \nu}} O\left(\lambda^{-n-3 / 2}\right) .
\end{aligned}
$$

Since $v_{k}=v_{i}$ if $k \equiv j(\bmod 4)$, the relations (9.5) always suffice to give the values of the Wronskians in (13.3). The constants $C_{j}$ are defined by (8.9a).

The indices $l$ and $m$ for which the formulas (13.1) and (13.3) hold depend upon $\arg v$; hence, the pairs of linearly independent solutions of the given equation whose behavior can be determined over all of $\mathscr{D}_{z}$ depend upon $\arg v$. The 
selection of these fundamental pairs of solutions may be made using Theorem 1 and the connection formulas (13.1). We omit the details of the selection and state only the results.

Theorem 2. For each value of $\lambda$ such that $|\arg \lambda| \leqq \pi$, the given equation (2.5) has a pair of linearly independent solutions whose asymptotic behavior over all of $\mathscr{D}_{i}$ may be found from Theorem 1 and the connection formulas (13.1); namely.

$$
\text { if } \left.\begin{array}{c}
0 \leqq \arg v<\pi \\
\pi \leqq \arg v \leqq \pi+\varepsilon \\
-\pi<\arg \nu \leqq 0 \\
-\pi-\varepsilon \leqq \arg v \leqq-\pi
\end{array}\right\} \text { the fundamental pair is }\left\{\begin{array}{l}
u_{-2}, u_{1} \\
u_{-3}, u_{0} \\
u_{-1}, u_{2} \\
u_{0}, u_{3} .
\end{array}\right.
$$

14. Characteristic solutions. If $\frac{1}{2}(\sigma \nu-1)=k$, a non-negative integer,

$$
y_{m}(x, v)=e^{-\frac{1}{2}(2 k+1) x^{2}} H_{k}(\sqrt{4 k+2} x) .
$$

where $y_{m}(x, y)$ is a solution (6.1) of the transformed Weber equation (8.1) and $H_{k}(x)$ is the Hermite polynomial of order $k$. The Hermite polynomials are even or odd according to the parity of $k$ so that when $\frac{1}{2}(\sigma \nu-1)=k$,

$$
y_{m}(x, v)=(-1)^{k} y_{m \pm 2}(x, v) .
$$

These facts are reflected in the behavior of the solutions of the given equation. If $|\arg \lambda|<\varepsilon$,

$$
\mathscr{W}\left(u_{0}, u_{ \pm 2}\right)=\mathscr{W}\left(y_{0}, y_{ \pm 2}\right)+y^{(v-f) / 2} e^{-\frac{1}{2} \nu} e^{\dot{\doteq} \nu \pi i}\left[O\left(\lambda^{-n-1}\right)-O\left(\lambda^{-n-1}\right)\right] .
$$

where the upper or lower signs are to be used according as $\arg \nu<0$ or $\arg v>0$. When $v=2 k+1, \mathscr{W}\left(y_{0}, y_{ \pm 2}\right)=0$. Thus, as shown by LANGER [4], for certain values of $\lambda$ the Wronskian $\mathscr{F}\left(u_{0}, u_{ \pm 2}\right)$ vanishes. These characteristic values are countable in number and are given by the formula

$$
\lambda_{k}=2 k+1+O\left(\lambda_{k}^{-n-1}\right) .
$$

At the characteristic values,

$$
u_{0}\left(z, \lambda_{k}\right)=(-1)^{k} u_{ \pm 2}\left(z, \lambda_{k}\right)\left[1+O\left(\lambda_{k}^{-n-1}\right)\right],
$$

where the upper or lower signs are to be used according as $\arg \left(\nu\left(\lambda_{k}\right)\right)$ is negative or positive. We call $u_{0}\left(z, \lambda_{k}\right)$ a characteristic solution of the given equation. We can observe the unique behavior of a characteristic solution $u_{0}\left(z, \lambda_{k}\right)$ for real $z$ by referring to Theorem 1 .

Since $\left|\Re\left(\lambda_{k} \Phi\right)\right|<N$ for values of $z$ such that $-1 \leqq \mathfrak{\Re} z \leqq 1$ and $|\Im z|<N\left|\lambda_{k}\right|^{-1}$, a characteristic solution is oscillatory for $z$ in a strip of width $O\left(\lambda_{k}^{-1}\right)$ centered on the interval $[-1,1]$. In certain regions outside of such a strip, a characteristic solution is subdominant and decreases exponentially to zero as $|z| \rightarrow \infty$. These regions are sectors of width $\frac{1}{2} \pi-\varepsilon$ with vertices at $z= \pm \sqrt{\lambda_{k} / \nu\left(\lambda_{k}\right)}$ and which are centered on the real axis. In particular, a characteristic solution is uniformly bounded for real $z$. Only characteristic solutions and their multiples have this property. 


\section{References}

[1] Buснноцz, H.: Die konfluente hypergeometrische Funktion. Berlin-GöttingenHeidelberg: Springer 1953.

[2] Cherry, T. M.: Uniform asymptotic formulae for functions with transition points. Trans. Amer. Math. Soc. 68, 224-257 (1950).

[3] ERdÉlyi, A., M. Kennedy \& J. McGregor: Parabolic cylinder functions of large order. J. of Rational Mech. and Anal. 3, 459-485 (1954).

[4] LANGER, R. E.: The asymptotic solutions of a linear differential equation of the second order with two turning points. Trans. Amer. Math. Soc., forthcoming.

[5] LANGER, R. E.: The asymptotic solutions of ordinary linear differential equations of the second order, with special reference to a turning point. Trans. Amer. Math. Soc. 67, $461-490$ (1949).

[6] McKelvey, R. W.: The solutions of second order linear ordinary differential equations about a turning point of order two. Trans. Amer. Math. Soc. 79, 103-123 (1955).

The University of Michigan

Ann Arbor

(Received June 10, 1958) 